\title{
Modern methods of diagnosing addiction to psychoactive substances: neurophysiological aspects
}

\author{
Valentina B. Salakhova ${ }^{1}$, Darya B. Belinskaya ${ }^{2}$, Maria A. Erofeeva ${ }^{3}$, Irina V. Ulyanova ${ }^{3}$, Larisa E. Zotova ${ }^{4}$, Rina S. Khammatova ${ }^{5}$, \\ Olga V. Mizonova ${ }^{6}$
}

\begin{abstract}
Objective: The study of the features of the higher mental functions of the brain in patients suffering from addiction to psychoactive substances is very important for narcology. It is necessary not only to uncover the mechanisms of addiction, a complete understanding of the neurophysiological processes that determine the nature of the development and severity of the disease, but also to solve practical problems related to the development of treatment methods and the implementation of preventive measures.

Method: In the study of patients, a specially designed map was used containing information on the hereditary and demographic characteristics, on the course of the disease, as well as on the socio-cultural status of patients. To determine the functional state of cortical activity in the process of perception of unconscious signals, a special technique was used. The obtained results were subjected to dispersive analysis and the significance of differences was calculated according to Student's t-criterion.

Results: An analysis of premorbid personality traits made it possible to establish the prevalence of instability, pathological conformity, and hysteroidal features in patients. It should be noted that a large percentage of the surveyed had a hereditary burden of alcoholism, mainly in the line of the father. Curiosity, passive submission, imitation of friends were among the motives of the first drug use. All patients in the process of addiction formed the following features: increased excitability, increasing effective disorders (depression, dysphoria), aggravated by exacerbation of craving for the drug, a decline in morals and ethics (egoism, deceit, indifference to one's fate, hedonism, loss of sense of duty), disorders of the emotional-volitional sphere (emotional instability, hypersensitivity, vulnerability).

Conclusion: The analysis of the duration of latent periods and magnitude of the amplitudes of the late positive R3OO wave in patients with heroin addiction revealed clear differences between the data obtained upon presentation of an unconscious neutral and motivationally significant word Patients who use psychoactive substances, using the method of recording induced potentials of the brain, which is a sensitive indicator of the activity of brain structures in relation to the substance used, revealed a clear diffuse activation of the cerebral cortex in response to an unconscious verbal stimulus related to the motivation prevailing in patients, in particular to "heroin". Such activation, as well as the difficult dying out of the developed reactions to an unconscious stimulus, indicate certain neurophysiological foundations of the mechanism underlying the changes in the motivationa sphere and the stability of the pathological craving for the psychoactive substance.
\end{abstract}

Keywords: psychoactive substances, neurophysiological mechanisms, motives of drug use, heroin addiction

\section{INTRODUCTION}

In life, a multitude of external stimuli that form associations with one another, temporary connections act upon a person constantly. As a result, stimuli simultaneously or in a certain sequence affect the sense organs that lead to temporary changes in the excitability of individual analyzers. At the same time, such functional relationships can be created in the central nervous system, when the body's reactions to a part of external stimuli are carried out at the "unconscious level", without realizing them. Unconscious reactions occur in neurotic states and a number of other diseases. It is known, for example, that with the use of psychoactive substances (PAS), about $60 \%$ of temporary connections are formed at an unconscious level. Reactions developed by unconscious emotional signals are usually fixed

\footnotetext{
1 Ulyanovsk State University, Ulyanovsk, Russia.

2 Moscow State University of the Civil Engineering, Moscow, Russia.

3 Moscow University of the Ministry of Internal Affairs of Russia Named after V.Y. Kikot, Moscow, Russia.

4 Moscow Region State University, Moscow, Russia.

5 Sechenov First Moscow State Medical University, Moscow, Russia.

6 Ogarev Mordovia State University, Saransk, Russia.
}

\section{Correspondence: Valentina B. Salakhova \\ Ulyanovsk State University, Ulyanovsk, Russia. \\ E-mail:valentina_nauka@mail.ru}

Received: 22 Jul 2018, Accepted: 23 Oct 2018

(C) 2018 by the authors; licensee Modestum Ltd., UK. This article is an open access article distributed under the terms and conditions of the Creative Commons Attribution License (http://creativecommons.org/licenses/by/4.0/). 
in a long-term memory, wane with difficulty and can persist for a long time. To comprehend the signal, it is not enough to process information in the corresponding projection and associative cortical zones. An assumption is made that there is an additional nerve mechanism that ensures this process. Such a mechanism should integrate the neural activity of various parts of the cerebral cortex and subcortical structures, which would ensure the conscious perception of environmental stimuli. A mild sensory stimulus may cause activation of cortical neurons, but the space-time parameters of this excitation may not be sufficient for the stimulus to be realized. According to the existing opinion, this fact can serve as a physiological basis for the so-called unconscious perception (1).

Medical and biological studies conducted in a narcological clinic, indicate systemic disorders underlying persistent mental and physical dependence on psychoactive substances. At present, it is already possible and necessary to talk about drug addiction as an extremely complex disease, determined by a pathological craving for drugs, the development of a dysfunctional state when they are stopped to be used, and in extreme cases, mental degradation and persistent somato-neurological disorders that have a specific biological basis.

It has been shown that a person with this kind of intoxication first of all has disorders in the cognitive sphere, accompanied by disorders of attention, memory, perception processes, etc. $(2,3,4)$. The importance of studying the effect of PAS on higher cortical functions also lies in the fact that under contemporary life conditions, a person requires a high level of attention, quick decision making, and a fairly high quality of processing large information streams. However, it is well known that narcotic substances act primarily on these functions. Therefore, the study of the features of the higher mental functions of the brain in patients suffering from addiction to PAS, is very important for narcology. It is necessary not only to uncover the mechanisms of the dependence formation, a more complete understanding of the neurophysiological processes that determine the nature of the development and severity of the disease, but also to solve practical problems related to the development of treatment methods and the implementation of preventive measures $(5-11)$.

\section{MATERIALS AND METHODS}

One hundred patients with heroin addiction were examined - men aged 16 to 21, 2-3 weeks after the last use of the drug, when acute manifestations of the withdrawal syndrome were arrested, i.e. in the period of "post-abstinent state" (12). The subjects did not have pronounced somatoneurological pathology and associated mental illness. The duration of their addiction ranged from 6 months to 3 years (on average 1.5 years).

While examining patients, a specially designed map was used containing information on the hereditary and demographic characteristics, on the course of the disease, as well as on the socio-cultural status of patients. The analysis of the premorbid personality traits made it possible to establish the prevalence of instability, pathological conformity, and hysteroidal features in patients. It should be noted that a large percentage of the patients surveyed had a hereditary burden of alcoholism, mainly in the line of the father. Curiosity, passive submission, an imitation of friends were among the motives of the first drug use. In the process of drug addiction all patients formed the following features: increased excitability, increasing effective disorders (depression, dysphoria), aggravated by exacerbation of craving for the drug, a decline in morals and ethics (egoism, deceit, indifference to one's own fate, hedonism, the loss of sense of duty), disorders of the emotional-volitional sphere (emotional instability, hypersensitivity, vulnerability). A special technique was used to determine the functional state of cortical activity in the process of perception of unconscious signals. On the screen, located at a distance of $180 \mathrm{~cm}$ from the eyes of the subject, the words: common nouns and motivationally significant "heroin", associated with the patient's experiences, were shown. The display time is $15 \mathrm{~ms}$.

The subjects during the experiment recline with eyes open in a chair. Words appear alternately, in a random order, into different halves of the field of view to the right and left of the fixation point located in the center of the screen. Due to the fact that the display of the word is very short, it is perceived by the subjects as a dim flash. The adaptation process to darkness lasts 5-7 minutes. Before the study begins, the following instruction is given: "You have to look at the luminous point during the entire study. Periodically, words will appear to the right and left of it. You need to read and count them. Try not to turn your head".

Each study procedure includes two trials, the interval between which takes about 3 minutes. In the first test, induced potentials are recorded only for neutral words, in the second - for a neutral and motivationally significant word - "heroin", the place of presentation of which in the left and right fields of view was changed in each experiment. The responses generated by the cortex were averaged separately for motivationally significant and neutral words. The discharge electrodes were placed on the occipital $(01,02)$, central $(C 3, C 4)$, as well as left and right associative areas (A1, A2) - in 


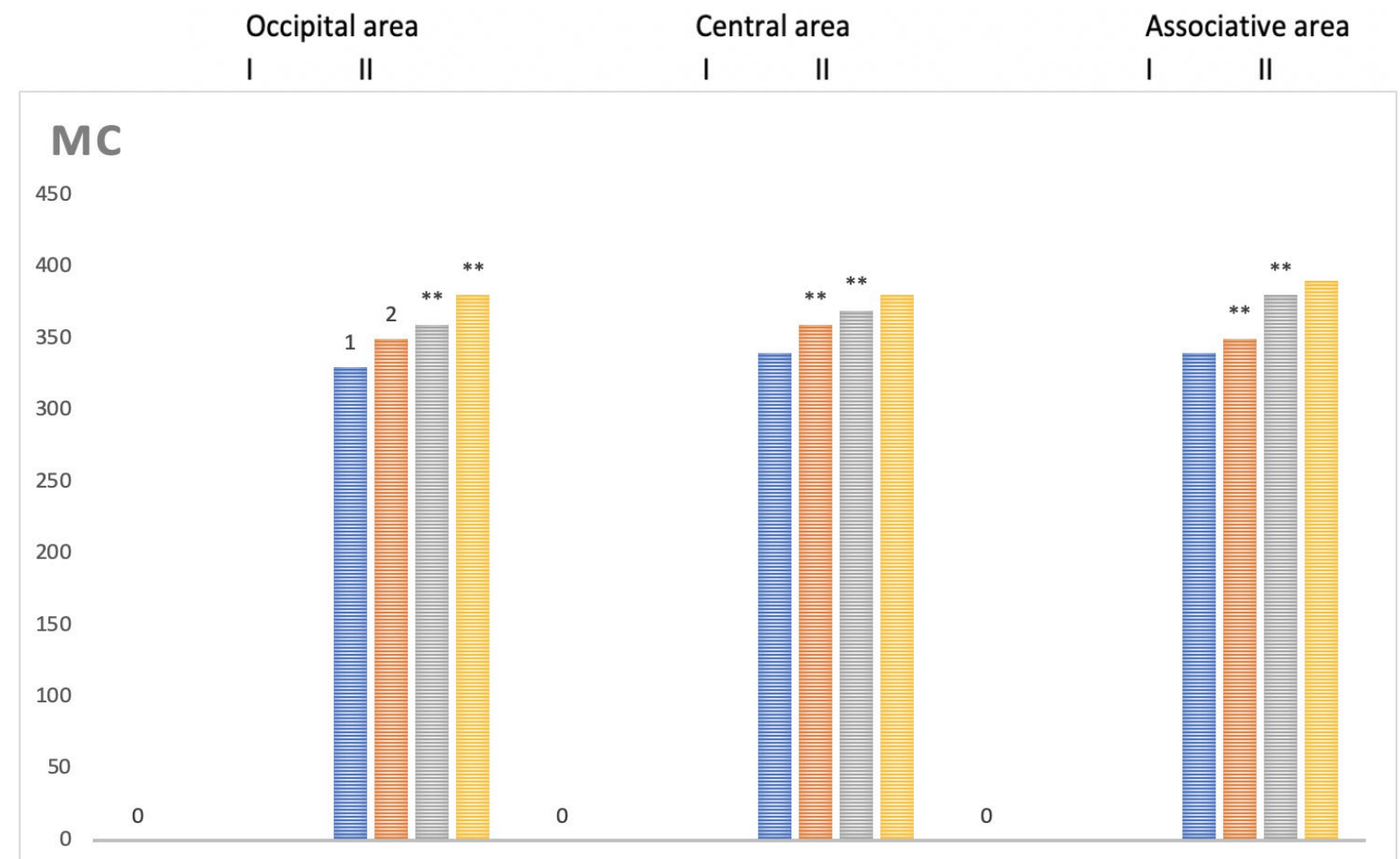

Figure 1: The latent period of the P300 wave in response to neutral (I) and motivationally significant (II) unconscious words in patients with heroin addiction and the left (1) and right (2) occipital, central and associative areas of the cerebral cortex

the middle of the distance between Pz (vertex) and the mastoid bone. Reference electrodes were placed on the left and right mastoid bones.

The latent period and the amplitude of the late positive P300 wave were automatically measured after the experimenter determined the peaks of the investigated wave and the peak of the previous oscillation on the graphic display. The obtained results were subjected to dispersive analysis and the significance of differences was computed according to Student's t-criterion. In all series of studies, the induced responses were compared to neutral words and to the motivationally significant word "heroin", as well as between the studied areas of the right and left hemispheres of the brain.

In addition, a psychological examination was carried out, which made it possible to identify the personality characteristics of patients, disorders in their motivational and emotional-volitional spheres, as well as the intellectualmnemonic level using modern methods of research. Critical and purposeful thinking, the consistency of judgments, and the ability of the patient to distinguish the essential signs of objects and phenomena were determined. The volume and stability of attention, memory were assessed.

\section{RESULTS}

The analysis of the duration of latent periods and the magnitude of amplitudes of the late positive P300 wave in patients with heroin addiction revealed clear differences between the data obtained upon presentation of an unconscious neutral and motivationally significant word.

The P300 wave with a latent period of $330.0+/-4.0 \mathrm{~ms}$ is set off to an unconscious neutral word from the left occipital area and on the right side $-350.0+/-4.3 \mathrm{~ms}$, i.e., there is a significant lengthening of the latent period of the studied wave in the occipital area of the right hemisphere relative to its magnitude in the left hemisphere $(F=10$, with $p<0.01)$.

The assessment of the duration of the P300 wave latent period, in response to an unconscious neutral word recorded in the central regions of the left and right hemispheres, also showed its significant lengthening in the right hemisphere $(340.0+/-4.0 \mathrm{~ms}$. and $360.0+/-4,0 \mathrm{~ms}$, respectively) $(\mathrm{F}=15 ; \mathrm{p}<0.01)$.

An analysis of the P300 wave latent period in response to a neutral word registered in the associative areas showed that in the associative area of the left hemisphere it was equal to $340.0+/-4.5 \mathrm{~ms}$, and in the right associative region $370.0+/-4.5 \mathrm{~ms}(\mathrm{~F}=17 ; \mathrm{p}<0.01)$, i.e. a significant increase in the latency of the late positive wave in the right hemisphere was again found (Figure 1). 


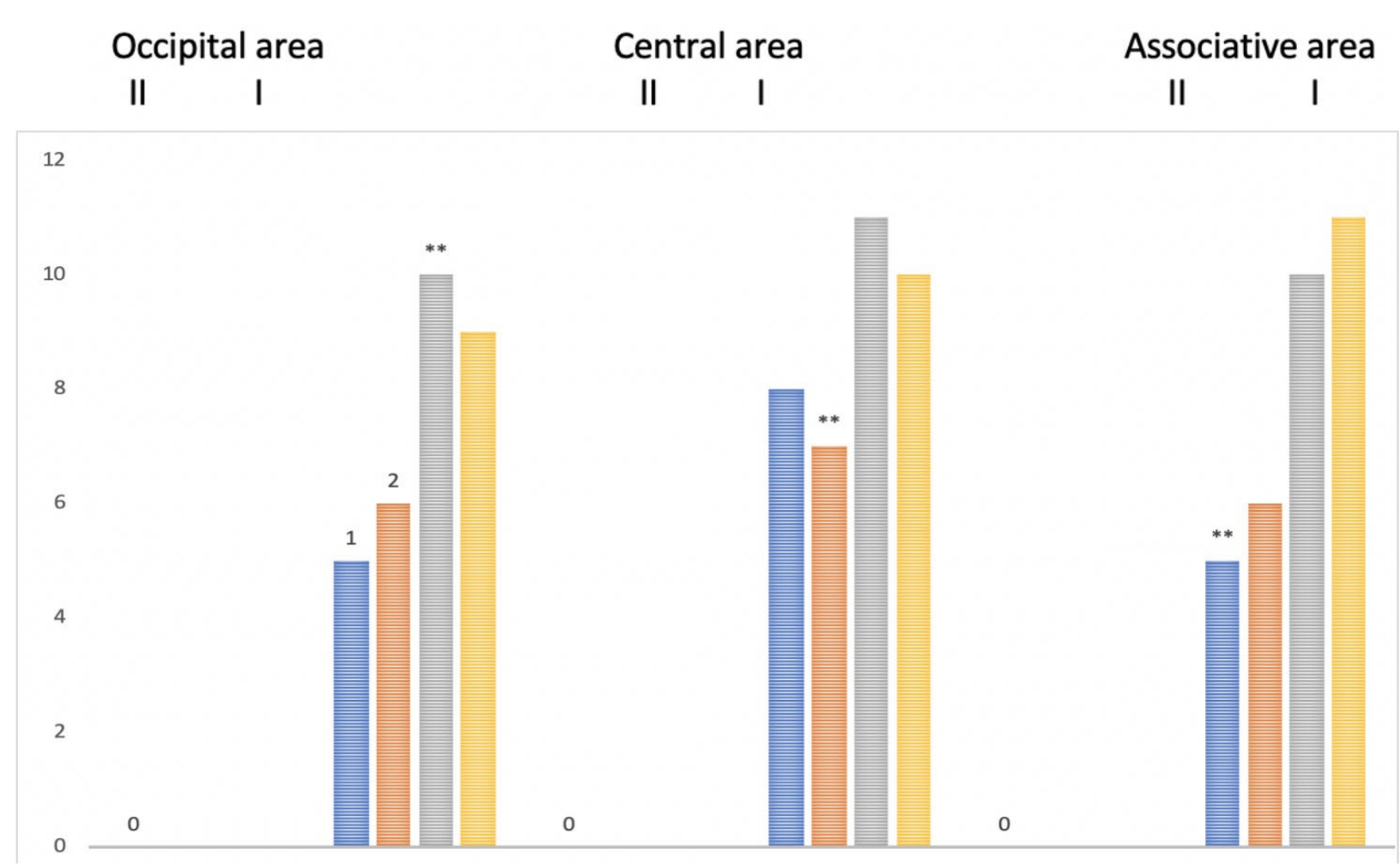

Figure 2: $P 300$ wave amplitude in response to neutral (I) and motivational-significant (II) unconscious words in patients with heroin addiction in the left (I) and right (II) occipital, central and associative areas of the cerebral cortex

When assessing the response of the studied wave when a neutral stimulus was presented in different fields of view, it was found that the P300 wave is recorded in the left occipital area, the amplitude of which was $6.3+/-0.3 \mu \mathrm{V}$, and in the right $-6.9+/-0,2 \mathrm{mkv}$. Significant differences in the magnitude of the response between the right and left hemispheres were not found.

In the central areas of the cortex of the left and right hemispheres, it was found that the P300 wave in response to the neutral word on the left was $7.4+/-0.3 \mu \mathrm{V}$, and on the right $-7.1+/-0.3 \mu \mathrm{V}$, i.e. there were also no significant differences in the magnitude of the response between the right and left hemispheres.

As for the response received upon presentation of a neutral unconscious word, its magnitude in the associative area of the left hemisphere was $6.3+/-0.3 \mu \mathrm{V}$, and in the associative area of the right hemisphere $-6.6+/-0.3 \mu \mathrm{V}$. Significant differences between the right and left hemispheres have not been found (Figure 2).

Upon presentation of the unconscious motivationally significant word "heroin", the P300 wave is recorded in the left occipital area with a latent period of $360.0+/-3.0 \mathrm{~ms}$, which is significantly longer than the latent period of the P300 wave recorded in the same area of the left hemisphere in response to a neutral word $(330.0+/-4.0 \mathrm{~ms} .-\mathrm{p}<0.01)$.

In response to an unconscious motivationally significant word in the right occipital area, the P300 wave has a latent period of $380.0+/-3.0 \mathrm{~ms}$, which also turned out to be significantly longer than the duration of the LP when a neutral word was presented $(350.0+/-4.3 \mathrm{~ms} .-\mathrm{p}<0.01)$.

When evaluating the latent period of the P300 wave recorded in the central areas of the left and right hemisphere, the same tendency is revealed as in the occipital areas: in the left hemisphere, the latent period of the motivationally significant word has a latent period of $363.0+/-4.0 \mathrm{~ms}$ that is significantly more than the neutral word $(340.0+/-4.0$ $\mathrm{ms} ; \mathrm{p}$ 0.0).

In the central area of the right hemisphere, the electrical response of the cortex is recorded for the word "heroin", the late positive wave of which developed with a latent period of $380.0+/-3.0 \mathrm{~ms}$, which also significantly differs from the response to the presentation of a neutral word $(360.0+/-4.0 \mathrm{~ms})$.

A similar relationship is observed in the associative areas of the left and right hemispheres. In the left associative area, the induced motivational word is recorded with a latent period of $380.0+/-5.0 \mathrm{~ms}$, and of a neutral word is $340.0+/-$ $5.9 \mathrm{~ms} ; \mathrm{p}$ 0.01. In the associative area of the right hemisphere, there is also a significant increase in the latent period of response to the motivationally significant word "heroin" relative to neutral, respectively: $395.0+/-4.5 \mathrm{~ms} ; 370.0+/-4.5$ ms: $p 0.01$. 
The data obtained show that there is a disorder of interhemispheric asymmetry in patients with heroin addiction. It manifests itself in the presentation of both a neutral and motivationally significant word: the latent period of the P300 wave is significantly extended in all recorded areas, but in the right hemisphere this elongation is more pronounced than in the left, and this effect is more pronounced when presenting a motivationally significant word (Figure 1).

The results of the evaluation of the potential of the wave under study, when presenting a motivationally significant word in different fields of view, showed that its amplitude in the left occipital area is $10.0+/-0.3 \mu \mathrm{V}$, and in the right $9.9+/-0.4 \mu \mathrm{V}$, i.e. the amplitude of this wave in response to a significant motivational word increases both in the left and in the right hemispheres compared to the amplitude obtained for the presentation of a neutral word in the same areas on the left and right $(6.3+/-0.3$ and $6.9+/-02 \mu \mathrm{V})$.

When analyzing the amplitude of the P300 wave, induced in response to a motivationally significant word that is recorded in the central areas of the left and right hemisphere cortex, it was found that in the left hemisphere it was 11.0 $+/-0.4 \mu \mathrm{V}$, and in the right hemisphere $-10,7+/-0.35 \mu \mathrm{V}$ (the differences are not significant). As for the magnitude of the P300 wave, obtained in response to the neutral word in the left and right central areas, their amplitude was $7.4+/$ $0.3 \mu \mathrm{V}$ and $7.1+/-0.3 \mu \mathrm{V}$, respectively (unreliable difference), that is the amplitude of this wave in response to a significant motivational word also increases in both the left and right hemispheres compared to the amplitude of the presentation of a neutral word.

In the left associative area in response to a motivationally significant word, the P300 wave is recorded with an amplitude of $10.7+/-0.4 \mu \mathrm{V}$, and on the right $-10.9+/-0.3 \mu \mathrm{V}$. Upon presentation of a neutral word from the left and right associative areas, potentials are recorded, the amplitudes of which were, respectively, $6.3+/-0.3$ and $6.6+/-0.2$ $\mu \mathrm{V}$, i.e. and in this case, the amplitude of this wave in response to a significant motivational word increases in both the left and right hemispheres compared to the presentation of a neutral word.

Thus, the influence of the emotional factor caused by an unconscious motivationally significant word $(F=16.58 ; p$ $<0.001$ ) is highly statistically significant in response to the word "heroin", an increase in the P300 wave is observed in all recorded areas of the cortex (Figure 2).

Psychological research revealed cognitive disorders in all patients with heroin addiction. There is a tendency to a decrease in intellectual abilities. Thinking was characterized by concreteness, which was expressed in the fact that patients established links between phenomena on formal grounds and were not capable of doing abstraction operations. For many of them, such mental operations as analysis, synthesis and generalization were difficult to cope with.

All patients taking PAS, there was a loss of interest in public life. They had a narrow range of interests - all thoughts focused on drugs. The primitiveness of judgments, sticking to the "trifles" were observed. In the conversation, the patients quickly got tired, often could not concentrate on, or closely pay attention to the content of the conversation. While testing their memory, there was a disorder of the dynamics of memorization and a decrease in direct and mediated memory. Torpid thinking, poor imagination were characteristic manifestations of intellectual-mnestic disorders.

Studying their emotional-volitional sphere, emotional instability and hypersensitivity were noted. Patients often demonstrated bad mood, melancholy, anger, tearfulness. Affective fluctuations persisted for a long time, with a predominance of periods of dysphoricity, irritability, irascibility, which gave way to apathy and sluggishness.

Thus, the results of a psychological study confirmed the above-described electrophysiological disorders in patients using PAS and showed that with their chronic intoxication, there are primarily disorders in the cognitive sphere, manifested in a weakening of intellectual and mnestic activity, moral and ethical degradation, disorders of the emotional sphere.

\section{DISCUSSION AND CONCLUSION}

Numerous studies indicate the possibility of perceiving the semantic properties of a word without its comprehending. Psychological studies on people with split brain hemispheres confirm this idea. Such people perceive individual words and react to them correctly, but without their determining. Experiments with the registration of evoked potentials of the cerebral cortex emphasize the same thing. It is very important, in our opinion, that such studies confirm the hypothesis expressed as far back as the 20-s by E.A. Kostandov (13) about the neurophysiological mechanism of the phenomenon of psychological defense. According to his point of view, raising the threshold of comprehension of the "emotional" word is due to the fact that it is still at the "preconscious level" perceived and "realized" by the cerebral cortex. An unconscious emotional verbal stimulus can also enliven temporary connections between the perceiving elements of the neocortex and the limbic system. Corticofugal excitation of the structures of the limbic system leads to the emergence of an 
ascending "nonspecific" impulsation, which through the reticular formation, and possibly directly, changes the activity of the cortical elements.

This identified feedback mechanism, due to which the cerebral cortex regulates the activity of its own neurons according to the signal value of the currently acting stimuli, is the possible basis for changing the thresholds of the emotional stimuli realization, in particular, verbal ones (14 - 19).

The studies conducted, especially with induced potentials on unconscious words, speak about the validity of the proposed hypothesis. In these works, important evidence was obtained that the emotional verbal stimulus, even before it was realized, could alter the activity of the cortical elements, which should also be reflected in the cortical mental functions.

The function of realizing an external stimulus, in this case a verbal one, and electrophysiological reactions to it, apparently, are carried out by different structural and functional levels that ambiguously change their activity under the influence of ascending impulses from the structures of the limbic-reticular complex.

It is believed that "the cortex is capable of perceiving peripheral stimuli without the appearance of an image, i.e. without the participation of sensory stellate neurons" (20). According to this interpretation, with mild, imperceptible stimuli, the netlike formation is not sufficiently activated, as a result of which the system of sensory stellate cortical neurons is not excited by these stimuli through specific paths $(12,21-24)$.

Considering the literature and the results of our study, as well as the data on the role of the limbic system in emotional processes, it can be assumed that these changes are reflected in the motivational sphere of patients with heroin addiction: in particular, they can play a certain role in the pathological craving for the drug.

The growth of the induced response to the motivational word "heroin" occurred in both hemispheres. At the same time, the smoothing of hemispheric ties was observed. Neurophysiological studies with alcohol intoxication using induced responses showed a significant decrease in the activity of the cerebral cortex, and to a large extent this decrease was manifested precisely in the area of the right hemisphere. Studies conducted on healthy people after drinking alcohol, as well as in patients with chronic alcoholism, also showed a lateralized effect of alcohol, manifested in greater suppression of the right hemisphere compared to the left (25-31).

Computer studies have revealed the presence of structural changes in the use of PAS, and clinical work has established the similarity of psychopathological syndromes with focal lesions of the right hemisphere with characteristic mental changes, such as complacency, euphoria, and a decrease in criticism, including to their condition $(32,33)$.

The above data indicates certain inter-hemispheric changes in the use of psychoactive substances. There are studies that have shown that in alcoholism, due to demyelination of peripheral nerves, partial sensory deprivation of the cortical ends of the analyzers can occur. The cortical activation revealed by us, apparently, can be one of the nervous mechanisms of a stable craving for alcohol, which is poorly corrected by the conscious experience of the patient.

Thus, in patients who use psychoactive substances, using the method of recording evoked potentials of the brain, which is a sensitive indicator of the activity of brain structures in relation to the substance used, a clear diffuse activation of the cerebral cortex has been revealed in response to an unconscious verbal stimulus related to the motivation prevailing in patients to "heroin" in particular. Such activation, as well as the difficult dying out of the developed reactions to an unconscious stimulus, indicate a certain neurophysiological mechanism underlying the changes in the motivational sphere and the sustainability of the pathological craving for a psychoactive substance.

\section{ACKNOWLEDGEMENTS}

The publication was prepared in the framework of the project supported by the Grant President, order number MK1562.2018 .6

\section{REFERENCES}

1. Ivanets NN, Vinnikova MA. Heroin addiction. Moscow: Medical practice. 2001.

2. Beritov IS. Structure and function of the cerebral cortex. Moscow: Science. 1969.

3. Erofeeva M, Khramtsova F. Social immunity youth. In Japanese Educational and Scientific Review. 2015;1(9):428436.

4. Klimenko TV. Addiction today. In Doctor. 1995;4:34-6.

5. Boelen P. Intolerance of uncertainty and emotional distress following the death of a loved one. In Anxiety Stress Coping. 2010;23(4):471-8. https://doi.org/10.1080/10615800903494135 PMid:20455126 
6. Kalinina NV, Salakhova VB, Artamonova EG, Efimova OI, Kalinin IV. Psychological Prevention Mechanisms of Minors' Deviant Behavior. Eurasian Journal of Analytical Chemistry. 2017;12(Special Issue):663-72. https://doi.org/10.12973/ejac.2017.00200a

7. Kozlov AA., Elder TC. Psychological features of drug addicts. Journal of Neurology and Psychiatry. 1999;10:14-9.

8. Makarov VV. Psychotherapy in narcology. Narcology. 2002;2:37-41.

9. Pyatnitskaya IN. Addiction. Moscow: Medicine. 1994.

10. Pyatnitskaya IN. Primary drug prevention. Narcology. 2002;8:19-25.

11. Wroblewsky AG. Medico-social prognosis of some forms of drug addiction. Questions of Addiction. 1988;3:3842.

12. Belcher HM. Substance abuse in children- prediction, protection, and prevention. Pediatr Adolesc. Med. 1998;152(10):952-60.

13. Kostandov EA. The perception of emotions. Moscow: Medicine. 1977.

14. Adelekan ML, Odejide OA. The reliability and validity of the WHO student drug-use questionnaire among Nigerian students. Drug and Alcohol Depend. 2002;24(3):245-9. https://doi.org/10.1016/0376-8716(89)90062-8

15. Dmitrieva TB, Drozdov FZ, Kogan BM. Clinical neurochemistry in psychiatry. Moscow: RIO SSC SSP them. V.P. Serbian. 1998.

16. Kaplan GI, Sadok BJ. Clinical Psychiatry: Moscow; 1994.

17. Krent D, Melhem N, Donohoe B, Walker M. The Incidence and Course of Depression in Bereaved Youth 21 Months after the Loss of a Parent to Suicide, Accident, or Sudden Natural Death. Am. Journal Psychiatry. 2009;166:786-94. https://doi.org/10.1176/appi.ajp.2009.08081244 PMid:19411367 PMCid:PMC2768496

18. Nikityuk DB, Miroshkin DV. Immune structures with increased physical exertion. Morphology. 2008;133(2):85-90.

19. Shcherbakova Ol, Chernykh AK, Balahanova DK, Midova VO. Stress control practices for mental balance improvement in academic sports. Teoriya i Praktika Fizicheskoy Kultury. 2018;9(1):22-3.

20. Aleksandrovsky YuA. Border mental disorders: a guide for doctors. Moscow: GEOTAR-Media. 2007.

21. Erofeeva M. Interaktion der sozialen Geschlechtsrolle. Eastern European Scientific Journal. 2014. Retrieved from http://www.auris-verlag.de/mediapool/99/990918/data/DOI_10.12851_EESJ201312.pdf

22. Jind L, Elklit $A$, Christiansen D. Cognitive schemata and processing among parents bereaved by infant death. Journal Clin. Psychol. Med. Settings. 2010;17(4):366-77. https://doi.org/10.1007/s10880-010-9216-1

23. Korolenko CP. Addictive behavior. General characteristics and patterns of development. Review of psychiatry and medical psychology. 1991;1:8-15.

24. Kuo JR, Linehan MM. Disentangling emotion processes in borderline personality disorder: physiological and selfreported assessment of biological vulnerability, baseline intensity, and reactivity to emotionally evocative stimuli. Journal Abnorm. Psychol. 2009;118:531-44. https://doi.org/10.1037/a0016392 PMid:19685950

25. Anokhin SP. The generality of the pathogenetic mechanisms of alcoholism and drug addiction and ways to find means to treat these diseases. Pharmacology and Toxicology. 1990;53(4):4-9.

26. Efimova OI, Salakhova VB, Mikhaylova IV, Gnedova SB, Chertushkina TA, Agadzhanova ER. Theoretical review of scientific approaches to understanding crisis psychology. Mediterranean Journal of Social Sciences. 2015;2:3-11.

27. Feldner MT, Monson CM, Friedman MJ. Critical Analysis of Approaches to Targeted PTSD Prevention: Current Status and Theoretically Derived Future Directions. Behav. Modif. 2007;31(1):80-116. https://doi.org/10.1177/0145445506295057 PMid:17179532

28. Klimenko TV. Social and biological causes of drug addiction. Doctor. 1992;12:33-5.

29. Krasnov VN. Affective spectrum disorders. Moscow: Practical medicine. 2011.

30. Nikityuk DB, Miroshkin DV, Bukavneva NS. Clinical and anthropological approaches. Morphological statements. 2008;1(2):56-62.

31. Shcherbakova OI. Formation managers stress management skills. Modern training and coaching: new opportunities in business and education. Moscow: Russian University of Economics. 2017.

32. Pyatnitskaya IN. Clinical Addiction Medicine. St. Petersburg: Medicine. 1975.

33. Salakhova VB, Kalinina NV, Belinskaya DB, Aygumova ZI, Tkhugo MM. Education as a factor of raising the adaptation potential in a delinquent personality. Man in India. 2017;97(3):1-13. 